\title{
A Remark on the Sloshing Frequencies for a Half-Space ${ }^{1}$
}

By B. Andreas Troesch, University of Southern California, Los Angeles, and Hans R. Troesch, University of Michigan, Ann Arbor, USA

\section{Introduction}

In a recent paper [4] the sloshing frequencies of an inviscid fluid in a half-space with circular or strip-like aperture have been investigated. The mathematical problem statement is of striking simplicity: the unknown velocity potential $\varphi$ satisfies the Laplace equation in a half-space, the normal derivative $\partial \varphi / \partial n$ vanishes at the boundary, except on the aperture where $\partial \varphi / \partial n=\lambda \varphi$ holds, and $\varphi$ decays sufficiently rapidly as the distance from the aperture increases.

Nevertheless, the numerical computation of the eigenvalues $\lambda$ requires, in addition to the general theoretical considerations, the rather extensive use of information on special functions of mathematical physics. It is the purpose of this note to show that the spectrum of the problem exhibits some noteworthy features, in accordance with the simplicity of the problem statement and in spite of the computational complications which are necessary to obtain the eigenvalues with rigorous error bounds.

Since the numerical results in [4] are remarkably accurate, it is feasible to deduce some properties of the spectrum on an empirical basis and then formulate the corresponding conjectures. In section 2 we will first state the known simple formulas for an upper and lower bound for an arbitrary eigenvalue. Then the next term in the asymptotic behavior is established in section 3 , and this result is justified theoretically.

The other question about the spectrum, considered in section 4, deals with the sum

$$
S=\sum_{n=1}^{\infty} 1 / \lambda_{n}^{2},
$$

evaluated separately for each symmetry class of the free oscillations. In the theory of integral equations the sum over the reciprocal squares of the eigenvalues equals the square of the kernel, integrated over the domain of all of its variables, provided appropriate conditions are satisfied (cf. [5], p. 141). The careful analysis of the numerical results in [4] suggests reasonable looking expressions for these sums; it would seem quite desirable to prove that these conjectures are indeed correct.

1) This research was supported by the National Science Foundation under Grant GP-22587. 


\section{Elementary Upper and Lower Bounds for All Eigenvalues}

Lower bounds for the $n$-th eigenvalue for a half-space with circular aperture are readily obtained by considering the eigenvalues for an infinitely deep cylinder of unit radius (cf. [4], p. 298):

$$
\lambda_{n, m}>j_{n, m}^{\prime} \quad n=1,2, \ldots, m=0,1,2 \ldots
$$

where $j_{n, m}^{\prime}$ is the $n$-the positive zero of the derivative of the Bessel function $J_{m}$. The integer $m$ represents the number of nodal diameters and designates the symmetry class.

On the other hand, simple upper bounds are furnished by the Rayleigh-Ritz method for the half-space with appropriate coordinate functions [6], namely

$$
\varphi_{n}= \begin{cases}J_{m}\left(j_{n, m} r\right) \exp \left(j_{n, m} z\right) \cos m \theta & \text { for } 0 \leq r \leq 1 \\ 0 & \text { for } 1 \leq r\end{cases}
$$

with $j_{n, m}$ denoting the positive zeros of $J_{m}$. These coordinate functions are admissible, and the matrices $A$ and $B$ in the eigenvalue equation

$$
\operatorname{det}(A-\lambda B)=0
$$

are both diagonal. Together with (2.1) it then follows that

$$
j_{n, m}^{\prime}<\lambda_{n, m}<j_{n, m}
$$

holds.

For the strip-like aperture (i.e., for two-dimensional or planar sloshing) the corresponding bounds are given by

$$
n \frac{\pi}{2}<\lambda_{n}<(n+1) \frac{\pi}{2}, \quad n=1,2, \ldots
$$

where in this context, the eigenvalues are numbered consecutively, and $n$ is odd for the antisymmetric, even for the symmetric planar mode.

\section{The Next Term in the Asymptotic Behavior}

From (2.2) and (2.3) it follows that all eigenvalues are confined to an interval which for the planar case is of length $\pi / 2$, and which tends asymptotically to $\pi / 2$ in the axially symmetric case. It is possible to make a more specific statement about the eventual position of the high eigenvalues within this interval. The eigenvalues have been reported up to $n=8$; and in the results due to Prof. G. H. Golub for $m=1$ even values for $n=40$ are still quite reliable (cf. [4], p. 314). Based on the trend of these numerical values, it becomes likely that for large $n$

$$
\lambda_{n, m}=\left(n+\frac{m}{2}-\frac{5}{8}\right) \pi+o(1)
$$


in the axially symmetric case, and

$$
\lambda_{n}=n \frac{\pi}{2}+\frac{\pi}{8}+o(1)
$$

in the planar case, the eigenvalues again numbered consecutively. This trend is shown as two examples in Figure 1 for $m=3$ and for the antisymmetric planar modes. The numerical results are listed in Tables 1,2 and 3 . It is observed that these asymp-

Figure 1

Difference $\delta_{3}$ from (3.1) for $m=3$, and difference $\delta_{\text {a.s. }}$ from (3.2) for the antisymmetric planar modes.

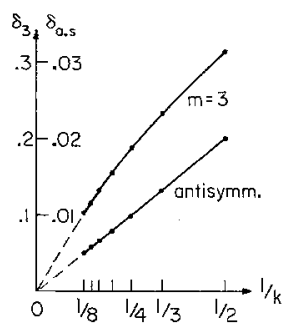

Table 1

The eigenvalues and their approximations, upper bounds $\lambda$ from [4], $\lambda^{*}$ from (3.1) and (3.2), $\lambda^{* *}$ from (3.4), $\lambda^{+}$from $\left(3.7^{\prime}\right)$.

\begin{tabular}{lcccccc} 
& $m=-\frac{1}{2}$ & $m=\frac{1}{2}$ & $m=1$ & $m=2$ & $m=3$ & $m=4$ \\
\hline$\lambda$ & 3.4533 & 2.0061 & 2.7548 & 4.1213 & 5.4001 & 6.6303 \\
$\lambda^{*}$ & 3.5343 & 1.9635 & 2.7489 & 4.3197 & 5.8905 & 7.4613 \\
$\lambda^{* *}$ & 3.4388 & 2.0165 & 2.7489 & 4.1075 & 5.4130 & 6.6973 \\
$\lambda^{+}$ & & 2.0033 & & & & \\
\hline$\lambda$ & 6.6286 & 5.1253 & 5.8922 & 7.3421 & 8.7184 & 10.0456 \\
$\lambda^{*}$ & 6.6759 & 5.1051 & 5.8905 & 7.4613 & 9.0321 & 10.6029 \\
$\lambda^{* *}$ & 6.6228 & 5.1278 & 5.8905 & 7.3340 & 8.7138 & 10.0572 \\
$\lambda^{+}$ & & 5.1250 & & & & \\
\hline$\lambda$ & 9.7839 & 8.2600 & 9.0329 & 10.5172 & 11.9408 & 13.3200 \\
$\lambda^{*}$ & 9.8175 & 8.2467 & 9.0321 & 10.6029 & 12.1737 & 13.7445 \\
$\lambda^{* *}$ & 9.7807 & 8.2611 & 9.0321 & 10.5119 & 11.9349 & 13.3201 \\
$\lambda^{*}$ & & 8.2599 & & & & \\
\hline$\lambda$ & 12.9330 & 11.3982 & 12.1743 & 13.6775 & 15.1295 & 16.5419 \\
$\lambda^{*}$ & 12.9591 & 11.3883 & 12.1737 & 13.7445 & 15.3153 & 16.8861 \\
$\lambda^{* *}$ & 12.9310 & 11.3989 & 12.1737 & 13.6737 & 15.1243 & 16.5388 \\
$\lambda^{+}$ & & 11.3982 & & & & \\
\hline$\lambda$ & 16.0794 & 14.5378 & 15.3158 & 16.8311 & 18.3022 & 19.7378 \\
$\lambda^{*}$ & 16.1007 & 14.5299 & 15.3153 & 16.8861 & 18.4569 & 20.0276 \\
$\lambda^{* *}$ & 16.0779 & 14.5382 & 15.3153 & 16.8282 & 18.2977 & 19.7338 \\
$\lambda^{+}$ & & 14.5378 & & & & \\
\hline$\lambda$ & 19.2243 & 17.6781 & 18.4574 & 19.9811 & 21.4659 & 22.9187 \\
$\lambda^{*}$ & 19.2422 & 17.6715 & 18.4569 & 20.0277 & 21.5984 & 23.1692 \\
$\lambda^{* *}$ & 19.2232 & 17.6784 & 18.4569 & 19.9787 & 21.4620 & 22.9146 \\
$\lambda^{*}$ & & 17.6781 & & & & \\
\hline & & & & & & \\
\hline
\end{tabular}


Table 2

High eigenvalues $\lambda$ for $m=1$ obtained by Prof. G. H. Golub, and approximation (3.1).

\begin{tabular}{llll} 
& $n=20$ & $n=30$ & $n=40$ \\
\hline$\left(n+\frac{m}{2}-\frac{5}{8}\right) \pi$ & 62.439154 & 93.855081 & 125.271007 \\
$\lambda$ & 62.439177 & 93.855098 & 125.271027
\end{tabular}

Table 3

Approximation of the eigenvalues $\lambda$ in the antisymmetric case by (3.2), $d_{n}=\lambda-\left(\frac{n}{2}+\frac{1}{8}\right) \pi$.

\begin{tabular}{llllllll}
\hline$n=1$ & 3 & 5 & 7 & 9 & 11 & 13 & 15 \\
\hline$d_{n}=0.0426$ & 0.0202 & 0.0133 & 0.0099 & 0.0079 & 0.0066 & 0.0057 & 0.0050 \\
\hline
\end{tabular}

totic results, called $\lambda^{*}$, approximate the eigenvalues quite reasonably, even for $n$ less than 6 . The asymptotic formula follows the type found for ordinary differential equations rather than that for partial differential equations (see [2], chap. VI), and this could have been anticipated from the nature of the problem.

It has been pointed out by Prof. J. B. Keller, that the asymptotic behavior of the eigenvalues can be deduced from the known solution of the dock problem [3]. For very short wave lengths, i.e., for high sloshing modes, the aperture appears so large in relation to the wave length that the phase of the wave at the rim is well approximated by the solution for a dock in an infinite ocean.

Let us first consider planar sloshing, since this represents the simpler case. The velocity potential $\varphi$ at the free surface, which is taken to be the positive $x$-axis, turns out to be (see [7], p. 323)

$$
\varphi(x, 0)=\cos \left(\lambda x-\frac{\pi}{8}\right)+o(1) .
$$

It then follows immediately (cf. Fig. 2a for an example) that for the half-width $a$ of the strip-like aperture

$$
2 a=\frac{n \pi}{\lambda}+\frac{\pi}{4 \lambda}
$$
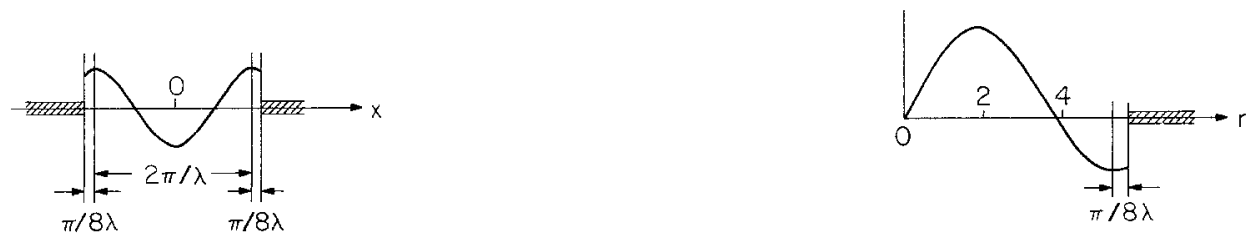

Figure 2

Examples for the phase relation near the rim, from the asymptotic formula (3.3). a) Symmetric planar case, b) axially symmetric case, $m=1$. 
holds, or for $a=1$

$$
\lambda \sim n \frac{\pi}{2}+\frac{\pi}{8}
$$

as asserted.

For the axially symmetric case we note that the $n$-th positive zero of the derivative $J_{m}^{\prime}$ of the Bessel function occurs asymptotically at

$$
j_{n, m}^{\prime} \sim(n-1) \pi+m \frac{\pi}{2}+\frac{\pi}{4}
$$

([1], chap. 9) for $m \geq 1$. (As observed in [4], p. 314, the spectrum for $m=0$ is the same as that for $m=2$ and will therefore not be considered further.) Thus, with a phase increment of $\pi / 8$,

$$
\lambda_{n, m}=n \pi+m \frac{\pi}{2}-\frac{5 \pi}{8}+o(1) .
$$

An example for $m=1$ is shown in Figure $2 \mathrm{~b}$. In this connection the question arises, why we have not applied the phase increment $\pi / 8$ to the true extrema of the Bessel functions rather than to their asymptotic extrema. This would indeed improve the approximation and reduce the error very substantially, except for $m=1$ and $m=2$. However, a closer inspection of the difference $\lambda-\lambda^{*}$ (cf. Table 1) reveals that their reciprocal values depend linearly on $n$, with the exception of $m=1$, where the dependence on $n$ is quadratic. This is an indication that for $m=1$ the next term in the asymptotic formula vanishes, so that the astonishingly good approximations given by $\lambda^{*}$ for $m=1$ are not purely accidental.

Admittedly, any set of smooth results will exhibit some trend, but the differences $\lambda-\lambda^{*}$ listed in Table 3 for the antisymmetric planar modes are just too suggestive to be ignored. The most likely asymptotic behavior is represented, to terms of order $1 / v^{2}$, by

$$
\lambda^{* *}=v-\frac{\pi}{8}-\frac{m(m-1)}{2 v}
$$

with

$$
v=\frac{\pi}{2}(2 k+m-1) .
$$

This formula is identical for the axially symmetric case $(m=1,2, \ldots)$, the symmetric plane case $\left(m=-\frac{1}{2}\right)$ and the antisymmetric plane case $\left(m=\frac{1}{2}\right)$. The latter two choices for $m$ are suggested by the considerations in [4], p. 300. For the symmetric plane case the trivial lowest eigenvalue $\lambda_{1}=0$ is omitted as is customary, so that we have here $k=2,3, \ldots$, but with the obvious shift in indexing used in Table 1 . The equation 
(3.4) is spelled out as

$$
\lambda_{k}^{* *}=\left(k+\frac{m}{2}\right) \pi-\frac{5 \pi}{8}-\frac{m(m-1)}{\pi(2 k+m-1)}, \quad m=1,2, \ldots
$$

for the axially symmetric case,

$$
\lambda_{k}^{* *}=k \pi-\frac{\pi}{8}-\frac{3}{4 \pi\left(2 k-\frac{1}{2}\right)}, \quad k=1,2, \ldots
$$

for the symmetric planar case,

$$
\lambda_{k}^{* *}=k \pi-\frac{3 \pi}{8}+\frac{1}{4 \pi\left(2 k-\frac{1}{2}\right)}, \quad k=1,2, \ldots
$$

for the antisymmetric planar case. The first two terms agree with (3.1) and (3.2) and are, as already mentioned, denoted by $\lambda^{*}$ in Table 1 .

Table 1 shows that $\lambda^{* *}$ approximates the numerical eigenvalues quite well. Although the general structure of the third term in $\lambda^{* *}$ is clearly indicated, it is of course possible that, for instance, the last term in (3.7) should actually read $1 / 8 \pi k$, i.e.,

$$
\lambda_{k}^{+}=k \pi-\frac{3 \pi}{8}+\frac{1}{8 \pi k} \quad k=1,2, \ldots
$$

This possibility, denoted by $\lambda^{+}$approximates the eigenvalues $\lambda$ even more closely (cf. Table 1), but then (3.4) would have to be modified slightly for $m=\frac{1}{2}$.

\section{The Sum of the Squares of the Reciprocal Eigenvalues}

The sums

$$
S=\sum 1 / \lambda_{n}^{2}
$$

can be determined quite accurately from the numerical results in [4] and from the asymptotic behavior derived in section 3 .

Let us recall first some known results about the roots of the Bessel functions. By comparing the quadratic terms in the power series expansion with the product representation of the Bessel functions (cf. [1], chap. 9) one obtains for the positive roots $\lambda$ of $J_{m}(\lambda)=0$

$$
\sum^{\infty} 1 / \lambda^{2}=\frac{1}{4(m+1)}
$$

and for the positive roots $\lambda$ of $J_{m}^{\prime}(\lambda)=0$

$$
\sum 1 / \lambda^{2}=\frac{(m+2)}{4(m+1) m} \quad \text { for } m \geq 1
$$


and

$$
\sum 1 / \lambda^{2}=\frac{1}{8} \quad \text { for } m=0 .
$$

This last result is obtained from

$$
J_{0}^{\prime}(\lambda)=-J_{1}(\lambda)
$$

The corresponding well known results for the planar case are

$$
\sum 1 / \lambda^{2}=\frac{1}{6}
$$

and

$$
\sum 1 / \lambda^{2}=\frac{1}{2}
$$

for the positive roots of $\sin \lambda=0$ and $\cos \lambda=0$, respectively. These results are used in Tables 4 and 5 for the upper bounds $\hat{\lambda}$ and the lower bounds $\grave{\lambda}$, in agreement with the remarks made in section 2 above. For $m=0$ and for the symmetric planar case, the lowest term in the $\hat{\lambda}$-sum must be subtracted, in order to retain consistency.

Table 4

Axially symmetric case, (4.1), (4.2), and (4.3), with $\hat{\lambda}$ an upper bound, $\breve{\lambda}$ a lower bound to the eigenvalue $\lambda$.

\begin{tabular}{llll}
\hline$m$ & $\sum \hat{\lambda}^{-2}$ & $\sum \lambda^{-2}$ & $\sum \check{\lambda}^{-2}$ \\
0 & $\frac{1}{4}-\frac{1}{j_{0}^{2}}$ & & \\
& $=0.077085$ & 0.11258 & $\frac{1}{8}$ \\
1 & $\frac{1}{8}$ & 0.20264 & $\frac{3}{8}$ \\
2 & $\frac{1}{12}$ & 0.11258 & $\frac{1}{6}$ \\
3 & $\frac{1}{16}$ & 0.07775 & $\frac{5}{48}$ \\
4 & $\frac{1}{20}$ & 0.05935 & $\frac{3}{40}$ \\
5 & $\frac{1}{24}$ & 0.04798 & $\frac{7}{120}$ \\
6 & $\frac{1}{28}$ & 0.04026 & $\frac{1}{21}$ \\
$\infty$ & $1 / 4(m+1)$ & $\left(\frac{m+1}{m}\right) / 4(m+1)$ & $\left(\frac{m+2}{m}\right) / 4(m+1)$ \\
\hline
\end{tabular}

Table 5

Planar case.

\begin{tabular}{llll}
\hline Mode & $\sum \hat{\lambda}^{-2}$ & $\sum \lambda^{-2}$ & $\sum \check{\lambda}^{-2}$ \\
Antisymmetric & $\frac{1}{6}$ & $\frac{1}{3}$ & $\frac{1}{2}$ \\
Symmetric & $\frac{1}{2}-\frac{4}{\pi^{2}}=0.09471$ & $\frac{1}{9}+\frac{1}{2 \pi^{2}}=0.144885$ & $\frac{1}{6}$ \\
\hline
\end{tabular}


The values for the sum of the reciprocal squares of the eigenvalues $\lambda$ in Table 4 were obtained by summing the results in [4] and using the Euler-Maclaurin formula ([1], chap. 23) for $n>8$. To at least the accuracy shown, these values are represented by

$$
\sum 1 / \lambda_{n}^{2}=\frac{2}{m \pi^{2}} S_{m}
$$

where

$$
s_{m}=\sum_{n=1}^{m}(2 n-1)^{-2}
$$

is a partial sum of

$$
\frac{\pi^{2}}{8}=\sum_{n=1}^{\infty}(2 n-1)^{-2}
$$

In terms of the digamma function $\Psi$ (cf. [1], chap. 6) we then obtain

$$
\sum_{n=1}^{\infty} 1 / \lambda_{n}^{2}=\frac{1}{2 m \pi^{2}}\left\{\Psi\left(\frac{1}{2}\right)-\Psi^{\prime}\left(m+\frac{1}{2}\right)\right\} \quad \text { for } m \geq 1
$$

Setting $m=\frac{1}{2}$, this formula also furnishes the result in Table 5 for the antisymmetric case, whereas for the symmetric case the conjectured closed form expression given in Table 5 is in agreement with the numerical value to better than five digits.

Although these results are highly suggestive, their validity has not been proved. As an example that peculiar numerical coincidences can indeed occur, we note that

$$
\sum_{n=1}^{\infty}\left(n-\frac{1}{8}\right)^{-2}=2.005741
$$

which resembles the fundamental eigenvalue for the antisymmetric case [4]

$$
2.005551<\lambda_{1}<2.006124
$$

Nevertheless, this must be judged a coincidence, even if the term $\frac{1}{8}$ is similar to the term in the asymptotic expansion (3.2) above.

\section{Acknowledgment}

The authors wish to express their gratitude to Professor G. H. Golub for making the extremely accurate results available, which were used in Table 2, and to Professor J.B. Keller for pointing out the relevance of the dock problem. 


\section{References}

[1] M. Abramowitz and I.A. Stegun, Handbook of Mathematical Functions (National Bureau of Standards, Washington, D.C. 1964).

[2] R. Courant und D. Hilbert, Methoden der Mathematischen Physik, Vol. 1, 2nd ed. (Julius Springer, Berlin 1931).

[3] K. O. Friedrichs and H. Lewy, The Dock Problem, Commun. pure appl. Math. 1, 135-148 (1948).

[4] P. Henrici, B.A. TroesCH and L. WuYTACK, Sloshing Frequencies for a Half-Space with Circular or Strip-Like Aperture, Z. angew. Math. Phys. 21, 285-318 (1970).

[5] S.G. Michlin, Vorlesungen über lineare Integralgleichungen (VEB Deutscher Verlag der Wissenschaften, Berlin 1962).

[6] N.N. Morsery and A.A. Petrov, The Calculation of Free Oscillations of a Liquid in a Motionless Container, Adv, appl. Mech. 9, 91-154 (1966).

[7] H. RuBin, The Dock of Finite Extent, Commun. pure appl. Math. 7, 317-344 (1954).

\section{Summary}

The known sloshing frequencies of an incompressible and inviscid fluid in a half-space with circular or strip-like aperture are investigated in some detail, based on previous results [4]. The first two terms in the asymptotic expansion for large eigenvalues are determined and a conjecture is enunciated for the general structure of the next term. The asymptotic results are listed in Table 1 and compared with the known frequencies.

Furthermore, the sum of the reciprocal squares of the eigenvalues is computed numerically for each symmetry class; the results suggest a conjecture about the exact value of these sums.

\section{Zusammenfassung}

Es werden die bekannten Eigenfrequenzen der Schwingungen einer idealen Flüssigkeit in einem Halbraum mit kreisförmiger oder streifenförmiger freier Oberfläche näher untersucht (siehe [4]). Die zwei ersten Glieder in der asymptotischen Entwicklung der Eigenfrequenzen werden hergeleitet und eine Vermutung über das nächste Glied aufgestellt (Abschnitt 2,3). Die asymptotischen Ergebnisse werden mit den bekannten Frequenzen in Tabelle 1 verglichen.

Für die verschiedenen Symmetrieklassen der Schwingungen werden die reziproken Quadratsummen der Eigenwerte numerisch bestimmt und eine Vermutung über den Wert dieser Ausdrücke ausgesprochen (Abschnitt 4).

(Received: April 18, 1972)

Varia - Miscellaneous - Divers

\section{5th Israel Annual Conference on Aviation and Astronautics, Haifa 1973}

The Fifteenth Israel Annual Conference on Aviation and Astronautics will be held on Wednesday, March 15th, 1973 in Tel Aviv and Haifa respectively. The program of the Conference will consist of original contributions in Aeronautics, Aviation, Astronautics, and related fields of engineering and science. Papers presented at the Conference are published by the Israel Journal of Technology and distributed to participants at the conference. For further particulars please write to: The Secretary, Organizing Committee, 15 th Israel Annual Conference on Aviation and Astronautics, c/o Dept. of Aeronautical Engineering, Technion, Haifa. 